\title{
Forecast Fxperiments with the NASA/GLA Stratospheric/Tropospheric Data Assimilation System
}

\author{
By Kenji Takano ${ }^{1}$, Wayman E. Baker, Eugenia Kalnay, David J. Lamich ${ }^{2}$, \\ Joan E. Rosenfield ${ }^{2}$ and Marvin A. Geller \\ Laboratory for Atmospheres, NASA/Goddard Space Flight Center, \\ Greenbelt, Maryland 20771 , U.S. A. \\ (Manuscript received 10 November 1986, in revised form 17 March 1987)
}

\begin{abstract}
For the first time, a four-dimensional stratospheric/tropospheric data assimilation system with a top analysis level at $0.4 \mathrm{mb}$ has been developed and used to produce physically consistent gridded analyses for the stratosphere as well as the troposphere for a period during the First GARP Global Experiment (FGGE) and Limb Infrared Monitor of the Stratosphere (LIMS) (November 1978-May 1979).

The system consists of a two-dimensional optimum interpolation analysis with 18 mandatory pressure levels and a 19 level fourth order stratospheric/tropospheric general circulation model with a horizontal resolution of $4^{\circ}$ (latitude) by $5^{\circ}$ (longitude) and a top at $0.3 \mathrm{mb}$. The system allows the utilization of stratospheric data including LIMS, Tiros-N retrievals, rocketsondes and vertical temperature profile radiometer (VTPR) soundings in addition to the other FGGE level $2 \mathrm{~b}$ data. These data are analyzed every six hours.

In order to examine the quality of the analyzed data forecast experiments starting from different analyses are performed for the period of the stratospheric sudden warming of late February 1979. The results indicate that by employing the present four-dimensional assimilation approach, the medium-range forecast skill for this event is improved.
\end{abstract}

\section{Introduction}

Stratospheric data analyses have been carried out at three operational centers: the European Centre for Medium Range Weather Forecasts (ECMWF), the British Meteorological Office (BMO), and the National Meteorological Center (NMC). However, none of these organizations have implemented a fully interactive stratospheric/tropospheric analysis /forecast procedure. The ECMWF assimilation system does not resolve well the stratosphere, and its highest analysis level is

${ }^{1}$ Courant Institute of Mathematical Sciences, New York University, New York, New York 10012, U.S. A.

2 Sigma Data Services Corp., Rockville, Maryland 20852, U.S. A.
$10 \mathrm{mb}$ (Bengtsson et al., 1982). The stratospheric assimilation at the $\mathrm{BMO}$ is performed with specified lower boundary conditions at either $100 \mathrm{mb}$ or $316 \mathrm{mb}$ obtained from the tropospheric analysis, so that there is no feedback to the tropospheric system. The NMC analysis uses the adjustment technique of Cressman (1959) with climatology as a background field.

At NASA/GLA (Goddard Space Flight Center's Laboratory for Atmospheres) we have developed a four-dimensional data assimilation system which incorporates the fully interactive procedure. Through the use of the system we can obtain physically consistent fields of both quantities that are analyzed, such as the mass field, as well as those not directly measured, such as the stratospheric 
winds, vertical velocity, and momentum and heat fluxes. These quantities could then be used to study the stratospheric general circulation without the need to assume geostrophic stratospheric winds as previously done in the works by Hartmann (1976), Smith (1983), Hamilton (1982), and Geller et al. (1983, 1984).

In this paper, the impact of the new analysis is investigated in terms of its effect on the forecast skill during the period of the stratospheric sudden warming observed in late February 1979. In Section 2, the GLA assimilation system is described and the results of forecast experiments are presented in Section 3. Summary and future plans are contained in Section 4.

\section{The data assimilation system}

The GLA stratospheric/tropospheric data assimilation system consists of an 18 level, multivariate, two-dimensional optimum interpolation (OI) analysis scheme with a top analysis level at $0.4 \mathrm{mb}$ and a 19 level fourth order stratospheric/tropospheric general circulation model (S/T-GCM) with a horizontal resolution of $4^{\circ}$ (latitude) by $5^{\circ}$ (longitude) and a top at $0.3 \mathrm{mb}$ (Takano and Chao: Chapter VI in Kalnay et al., 1983).

The 18 level OI analysis scheme is an expanded version of the 12 level GLA OI analysis scheme developed for the troposphere (Baker et al., 1987). In addition to the multivariate upper-air analysis, a multivariate surface analysis over the oceans (Bloom et al., 1984), which maintains an Ekman balance instead of the usual geostrophic balance, is also utilized.

The S/T-GCM coupled with the OI analysis scheme is an extended version of the 9 sigma level GLA fourth order GCM originally developed for the troposphere (see also Kalnay et al., 1983) so that the major features of two models are nearly identical. Both GCMs use quadratically conservative fourth order horizontal differencing on an unstaggered grid and similar parameterization of subgrid scale physical processes. The main differences are the vertical coordinate system, the vertical differencing and the radiation calculations. The S/T-GCM incorporates a

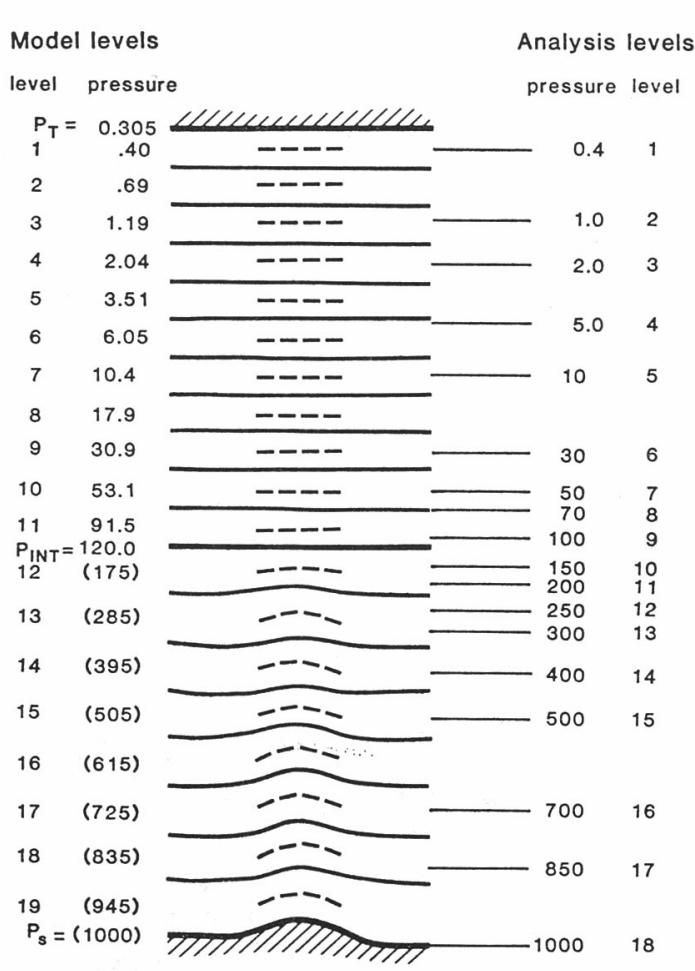

Fig. 1 Vertical structure for the stratospheric/ tropospheric data assimilation system. Pressure values with parentheses indicate sigma levels.

hybrid (sigma-pressure) coordinate system with 8 sigma and 11 pressure levels in the troposphere and the stratosphere, respective1y. These levels are designed to minimize interpolation between the GCM levels and the mandatory pressure levels used in the analysis scheme, and also the bottom 8 sigma levels are chosen to be similar to those of the original 9 sigma level GCM so that the tropospheric portions of both GCMs, including the physical processes, are nearly the same (Fig. 1). The vertical differencing is formulated in the manner suggested by Arakawa and Suarez (1983) for the sigma coordinates, and by Arakawa and Lamb (1977) and Tokioka (1978) for the pressure coordinates. The S/T-GCM incorporates a new radiative transfer algorithm as described in Rosenfield et al. (1987). The infrared radiative transfer is modeled based on the methods of Chou and Arking (1978, 1980) and Chou and Kouvaris (1981). The formulations of Lacis and Hansen (1974) and Ramanathan 
and Cess (1974) are used to estimate the solar radiative heating due to water vapor and carbon dioxide, respectively. The solar radiative heating due to ozone is estimated by the method of Strobel (1978) which is accurate and computationally efficient for varying ozone amounts (a desirable option in the GCM). The present version of the S/TGCM does not incorporate the dynamical and chemical processes of ozone. It uses the zonally averaged ozone mixing ratio for each month derived from Solar Backscatter Ultraviolet (SBUV) and Solar Mesospheric Explorer (SME) observations to estimate ultraviolet absorption and infrared radiative transfer due to ozone. Thus, the ozone data are not analyzed. In the future, the fully interactive radiative-chemical-dynamical processes of ozone will be incorporated into the assimilation system.

The meteorological data utilized in the system include LIMS, Tiros-N retrievals, rocketsondes and vertical temperature profile radiometer (VTPR) soundings in addition to the other FGGE level $2 \mathrm{~b}$ data. These data are analyzed every 6 hours. The LIMS data were provided by Dr. John C. Gille of NCAR.

\section{Forecast experiments}

In this section, the quality of the analyzed data will be examined by assessing the impact of the analysis used as initial conditions on the medium-range forecast skill. For this purpose, two 10-day forecast experiments starting from different analyses have been performed for the stratospheric sudden warming event observed in late February 1979.

The following two analyses at 17 February $12 Z$ were used as the initial conditions for the experiments. The first analysis was obtained by using the NMC analysis for the stratosphere and the GLA analysis for the troposphere (Baker, 1983). These two analyses were combined at $120 \mathrm{mb}$ and above this level the NMC daily analysis of temperatures was used to construct geopotential heights. Winds were derived geostrophically from the heights except in the tropics between $20^{\circ} \mathrm{N}$ and $20^{\circ} \mathrm{S}$ where the winds were obtained by linearly interpolating the geostrophic winds in latitude. Since the mass and momentum fields are not well balanced, especially in the tropics, these initial conditions result in a considerable amount of gravity wave activity during the early stage of the forecast. These initial conditions and the corresponding forecast will be referred to as "GLA/NMC".

The second was our analysis produced by a one-day assimilation. The first guess at 16 February $12 Z$ for the assimilation was created by the same method as that used for the GLA/NMC initial conditions. Unfortunately, the LIMS data were not available during the one-day assimilation. The initial conditions and the resulting forecast will be denoted as "OI". The assimilation was extended further to 27 February $12 Z$ and the last ten days' analyses are used as verification for the two forecast experiments.

First, we compare the forecast results for the mean zonal winds. Fig. 2 shows the GLA/NMC and the OI initial conditions for the mean zonal winds in the Northern Hemisphere created. Both initial conditions show three westerly jets: the polar night jet centered in the middle stratosphere, another jet at low latitudes with the maximum wind in the upper stratosphere/mesosphere region, and the subtropical jet in the tropopause region. There are, however, significant differences in the magnitude of these jets. The GLA/NMC initial conditions have stronger stratospheric jets but a weaker subtropical jet. The stronger jets in the GLA/NMC initial conditions are probably due to the use of the geostrophic approximation which overestimates the intensity of cyclonic circulation. The weaker tropopause subtropical jet may originate in the tropospheric GLA analysis which does not resolve the lower stratosphere properly.

In Fig. 3, comparisons of the mean zonal wind of the two forecasts are shown along with the OI analyses after four and ten days. After four days, both forecasts and the OI analysis exhibit easterlies in the polar regions associated with the sudden warming which have descended into the lower stratosphere, but the forecast polar easterlies, especially in the OI case, are weaker than in the verifi- 

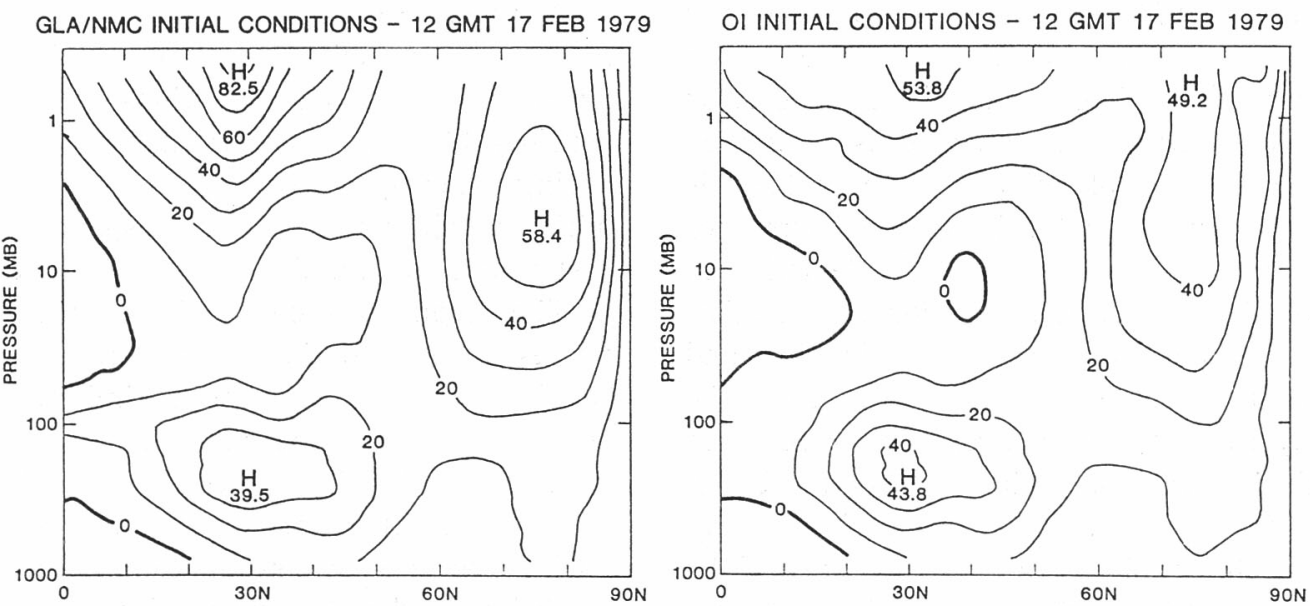

Fig. 2 Mean zonal winds $\left(\mathrm{ms}^{-1}\right)$ for 12 GMT 17 February 1979. The contour interval is $10 \mathrm{~ms}^{-1}$.
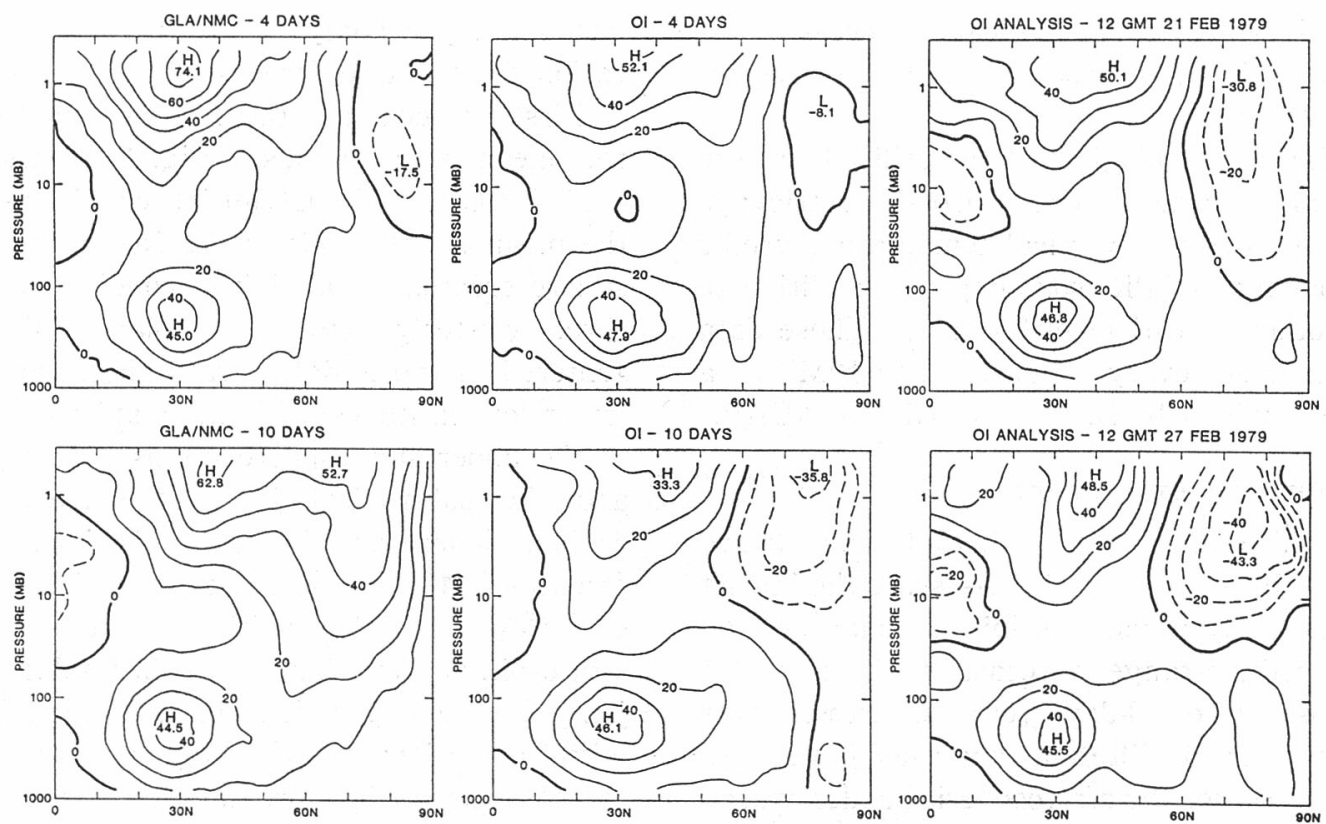

Fig. 3 Mean zonal winds $\left(\mathrm{ms}^{-1}\right)$ for 4 -day (top) and 10-day (bottom) forecasts from 17 February and the OI analyses.

cation. After ten days, both the OI analysis and the OI forecast have much stronger easterlies whereas the GLA/NMC forecast has no easterlies at all. The behavior of the stratospheric westerly jet is also better represented in the OI forecast, and the subtropical jet is well predicted in both experiments throughout the period.

The zonally averaged temperature changes from the initial conditions are next compared in Fig. 4. This quantity is a very sensitive measure of the forecast skill for the sudden warming event. The two initial analyses of the zonally averaged temperature cross section at 17 February $12 Z$ (not shown) are very similar except in the lower polar stratosphere where the OI analysis has a slightly higher temperature. Four days later, the OI analysis shows a warming in the polar region of about $25 \mathrm{~K}$ centered in the lower stratosphere 
with a cooling above of about $13 \mathrm{~K}$. In middle latitudes, there are also weak changes in the zonal temperature with a pattern which is reversed with respect to the polar changes: a weak cooling in the lower stratosphere and a weak warming in the upper stratosphere. The two forecasts at day four agree generally with the analyzed temperature change, with the GLA/NMC forecast showing stronger heating and cooling in the polar stratosphere. After ten days, the OI analysis shows a similar change in temperature to that seen after four days, but the magnitude of warming and cooling at the polar region is increased by about $10 \mathrm{~K}$. The OI forecast simulates the warming fairly well whereas the GLA/NMC forecast fails to maintain the warming. The cooling above the warming disappears from the OI forecast after ten days, while the GLA/NMC forecast exhibits too much cooling. It is noted that there is a difference in the height of maximum warming between the OI analysis and the ECMWF analysis. The height of about $30 \mathrm{mb}$ shown in the OI analysis (also in the NMC analysis) is lower than that of $10 \mathrm{mb}$ (the heighest analysis level) in the ECMWF analysis (Fig. 18 in Simmons and Strüfing, 1983). Further investigations will be needed to examine the effects of the upper boundary conditions on the forecast and the assimilation.

Finally, the forecast and analysis of geopotential height at $30 \mathrm{mb}$ are shown in Fig. 5 . For the initial conditions (17 February 12Z), there is a single elongated polar vortex extending from North America to Eurasia (not shown). After four days, the OI analysis depicts a split vortex, with one center over Siberia and the other over Canada, indicating the development of a wave number two warming (major warming) event. This split is predicted well in both forecasts with a slight overprediction of the depth of the lows in the GLA/NMC forecast. The Aleutian and Scandinavian highs have developed as observed in both forecasts. In the next six days, the OI analysis shows that the split vortex has rotated slightly clockwise, weakening the intensity of the two lows. The rotation and the maintenance of the split vortex are well reproduced in the OI forecast, although the lows are too intense. The GLA/NMC forecast, in contrast, results in an almost complete merging of the vortices, so
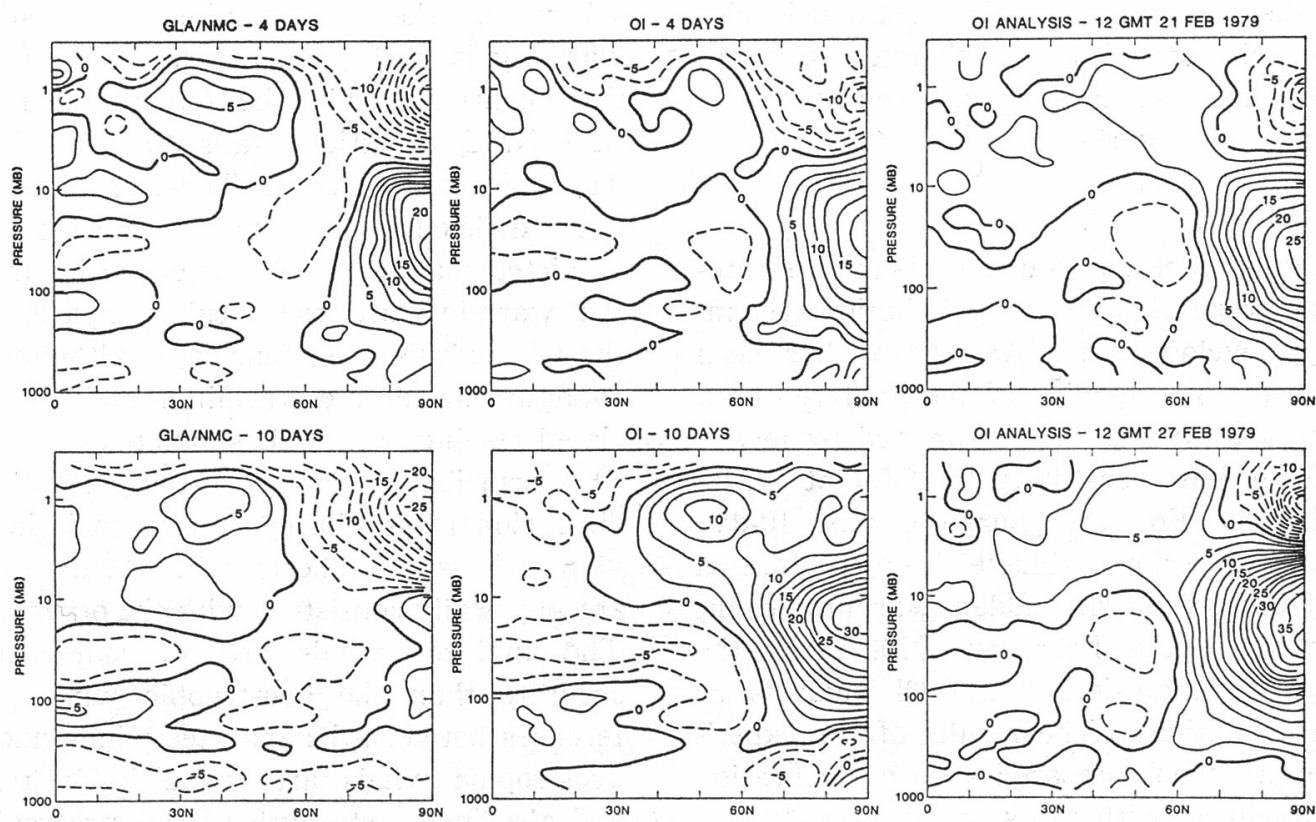

Fig. 4 Changes in zonally averaged temperatures (K) in the 4 days (top) and the 10 days (bottom) following 17 February. The contour interval is $2.5 \mathrm{~K}$. 

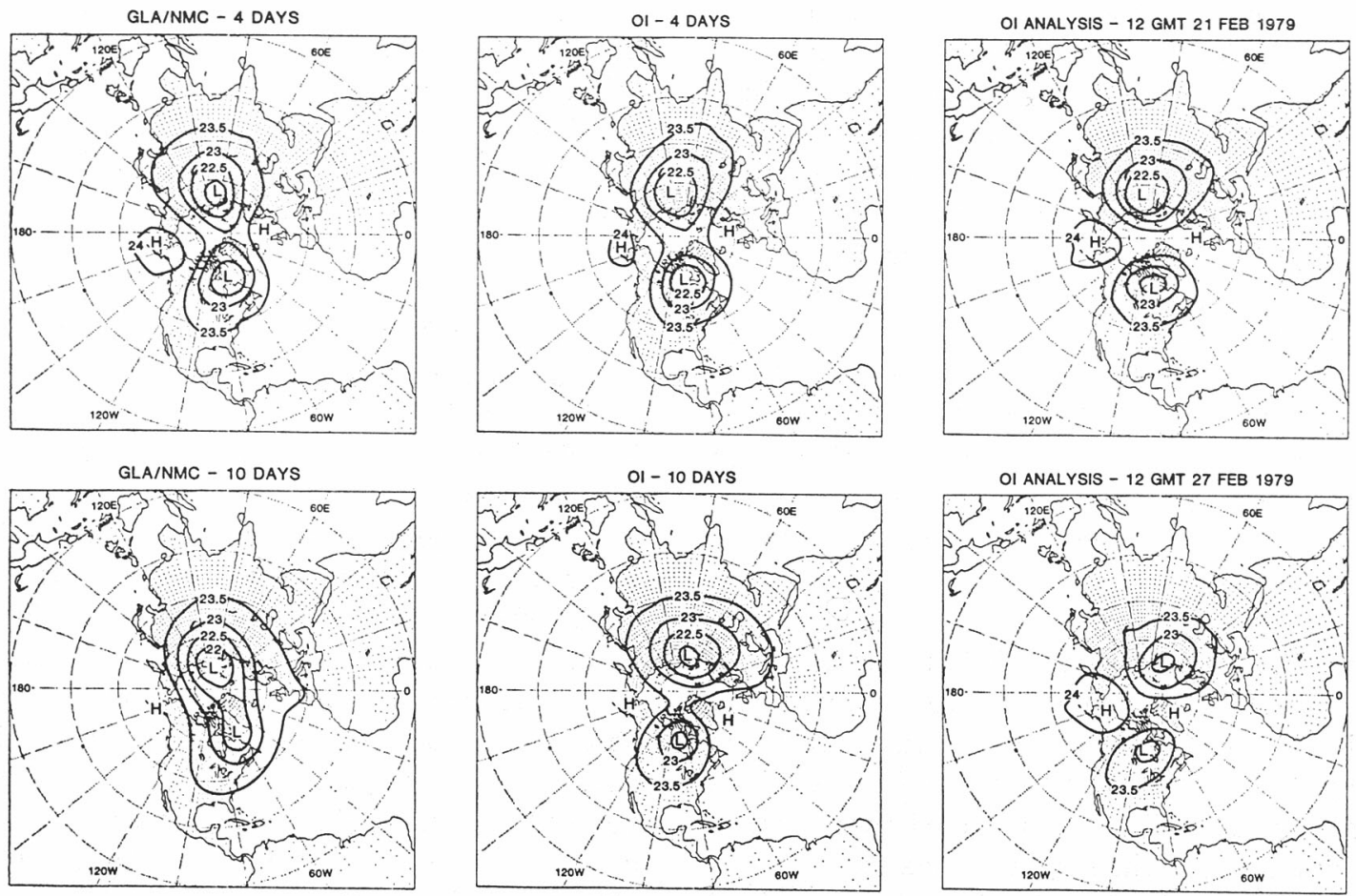

Fig. 5 The Northern Hemisphere maps of the geopotential heights ( $\mathrm{km}$ ) at $30 \mathrm{mb}$ for 4 -day (top) and 10-day (bottom) forecasts from 17 February and the OI analyses. The contour interval is $0.5 \mathrm{~km}$.

that the wavenumber two pattern is essentially disappeared. These forecast deficiencies are consistent with the changes in mean zonal wind and temperature shown in the previous two figures.

\section{Summary}

In this paper, a four-dimensional stratospheric/tropospheric data assimilation system, newly developed at NASA/GLA, has been described. The quality of the analyzed data by the system has been examined by means of the forecast sensitivity to different initial conditions. For this purpose, two 10-day forecast experiments have been performed for the stratospheric sudden warming event observed in late February 1979, using the GLA/NMC analysis and the OI analysis as initial conditions. The results of the experiments are then compared with a verification also produced with the system.

The results indicate that by using the OI analysis as initial conditions, the medium- range forecast skill is improved in the stratosphere for the case examined. The sudden warming is well predicted using the OI initial conditions, while the forecast from the GLA/NMC conditions fails to maintain the warming after ten days. There are, however, some deficiencies in the OI forecast. The predicted magnitude of the easterlies and of the warming are too weak compared with the OI analysis after four days, although the configuration of the warmings and the associated coolings are well simulated.

A principal advantage of the present fourdimensional assimilation approach is in providing the stratospheric wind fields, which are physically consistent with the other fields. The analyzed winds are, of course, more accurate than the geostrophic winds. Differences between the analyzed winds and the geostrophic winds are found in the tropics and also in the strength of the stratospheric jets. Thus, the OI analysis could be used to reestimate the stratospheric general circu- 
lation which has been previously determined from diagnostic studies based on the geostrophic assumption. As a next step, we plan to use the assimilation system to produce analyses of the entire period of the LIMS data (November 1978-May 1979) utilizing the other available FGGE data.

In the future, we plan to modify the OI analysis scheme and the S/T-GCM to permit an analysis of ozone mixing ratio as well as to develop the capability to use the direct wind observations that will be forthcoming from the Upper Atmosphere Research Satellite (UARS) scheduled to be launched by NASA in 1991.

\section{Acknowledgements}

Support for this research was provided by the NASA Upper Atmosphere Research Program. The technical assistance of Jack Woollen, Eugenia Brin and Herb Carus with the data assimilation system is gratefully acknowledged. Laura Rumburg drafted the figures.

\section{References}

Arakawa, A. and M. J. Suarez, 1983: Vertical differencing of the primitive equations in sigma coordinates. Mon. Wea. Rev., 111, 34-45.

- and V.R. Lamb, 1977: Computational design of the basic dynamical processes of the UCLA general circulation model. Methods in Computational Physics, 17, Academic Press, 173-265.

Baker, W.E., 1983: Objective analysis and assimilation of observational data from FGGE. Mon. Wea. Rev., 111, 328-342.

Baker, W.E., S.C. Bloom, J.S. Woollen, M.S. Nestler, E. Brin, T.W. Schlatter and G.W. Branstator, 1987: Experiments with a threedimensional statistical objective analysis scheme using FGGE data. Mon. Wea. Rev., 115, 272-296.

Bloom, S.C., W.E. Baker and M.S. Nestler, 1984: Multivariate optimum interpolation at surface pressure and winds over oceans. Preprints Tenth Conf. on Weather Forecasting and Analysis, 25-29 June, Clearwater, Florida, 106-108, Amer. Meteor. Soc.

Bengtsson, L., M. Kanamitsu, P. Kållberg and S. Uppala, 1982: FGGE 4-dimensional data assimilation at ECMWF. Bull. Amer. Meteor. Soc., 63, 29-43.

Chou, M.-D. and A. Arking, 1978: An infrared radiation routine for use in numerical atmospheric models. Reprint Volume of Third Con- ference on Atmospheric Radiation, 28-30 June, Davis, Calif.

and

1980: Computation of infrared cooling rate in the water vapor band. $J$. Atmos. Sci., 41, 1775-1778.

and L. Kouvaris, 1981: An efficient routine for infrared radiative transfer in a cloudy atmosphere. NASA Tech. Memo. 86064, NASA /Goddard Space Flight Center, Greenbelt, MD 20771.

Cressman, G.P., 1959: An operational objective analysis system. Mon. Wea. Rev., 85, 367-374.

Geller, M.A., M.F. Wu and M.E. Gelman, 1983: Troposphere-stratosphere (surface- $55 \mathrm{~km}$ ) monthly winter general circulation statistics for the Northern Hemisphere-four year average. $J$. Atmos. Sci., 40, 1334-1352.

stratosphere (surface-55 km) monthly winter general circulation statistics for the northern hemisphere-interannual variations. J. Atmos. Sci., 41, 1726-1744.

Hamilton, K., 1982: Some features of the climatology of the Northern Hemisphere stratosphere revealed by NMC upper atmosphere analysis. $J$. Atmos. Sci., 39, 2737-2749.

Hartman, D. L., 1976: The dynamical climatology of the stratosphere in the Southern Hemisphere during late winter 1973. J. Atmos. Sci., 33, 1789-1802.

Kalnay, E., R. Balgovind, W. Chao, D. Edelmann, J. Pfaendtner, L. Takacs and K. Takano, 1983: Documentation of the GLAS fourth order general circulation model. Volume I: Model documentation. NASA Tech. Memo. 86064, NASA/ Goddard Space Flight Center, Greenbelt, MD 20771, 404pp. (NTIS N8424028).

Lacis, A. A. and J.E. Hansen, 1974: A parameterization for the absorption of solar radiation in the earth's atmosphere. J. Atmos. Sci., 31, 118-133.

Ramanathan, V. and R.D. Cess, 1974: Radiative transfer within the mesospheres of Venus and Mars. Astrophys. J., 188, 407-416.

Rosenfield, J.E., M.R. Schoeberl and M.A. Geller, 1987: A computation of the stratospheric diabatic circulation using an accurate radiative transfer model. J. Atmos. Sci. in press.

Simmons, A. J. and R. Strüfing, 1983: Numerical forecast of stratospheric warming events using a model with a hybrid vertical coordinate. Quart. J. Roy. Meteor. Soc., 109, 81-111.

Smith, A.K., 1983: Stationary waves in the winter stratosphere: Seasonal and interannual variability. J. Atmos. Sci., 40, 245-261.

Strobel, D. F., 1978: Parameterization of the atmospheric heating rate from 15 to $120 \mathrm{~km}$ due to $\mathrm{O}_{2}$ and $\mathrm{O}_{3}$ absorption of solar radiation. $J$. Geophy. Res., 83, 6225-6230.

Tokioka, T., 1978: Some considerations on vertical differencing. J. Meteor. Soc. Japan, 56, 98-111. 